\title{
A NOTE ON EXPANSIVE MAPPINGS
}

\author{
RICHARD K. WILLIAMS
}

Let $f$ be a continuous multi-valued transformation of a metric space $X$ (with metric $d$ ) onto itself. For brevity, call $f$ a mapping.

If $x \in X$, an orbit of $x$ under $f$ is a set of the form

$$
\left\{x_{n}: x_{0}=x, x_{n+1} \in f\left(x_{n}\right) \text { for each integer } n\right\} \text {. }
$$

We say that $f$ is expansive on $X$ if there exists $\delta>0$ such that $x, y \in X, x \neq y$ implies that for each orbit $A$ of $x$ and for each orbit $B$ of $y$, there exist $x_{n} \in A, y_{n} \in B$ such that $d\left(x_{n}, y_{n}\right)>\delta$. (See [2].) The quantity $\delta$ is called an expansive constant for $f$. This generalizes the concept of expansive homeomorphism studied in [1], for instance.

It is known that if $f$ is a homeomorphism of $[0,1]$ onto itself, then $f$ is not expansive. (See [1].) The purpose of this paper is to show that $f$ is not expansive if it is a single-valued mapping $[0,1]$ onto itself, but that there are expansive mappings on $[0,1]$.

To show this, we need some preliminary results.

THEOREM 1. If $f$ is a single-valued, uniformly continuous mapping of $X$ onto itself, then $f$ is expansive if and only if $f^{n}$ is expansive for $n \neq 0$.

Proof. Since it is obvious that $f$ is expansive if and only if $f^{-1}$ is, let us assume that $n>0$. It is also clear that if $f^{n}$ is expansive, then $f$ is. Assume therefore that $f$ is expansive with expansive constant $\delta$. By uniform continuity, there exists $\Delta>0$ such that $d(x, y)<\Delta$ implies $d\left(f^{i}(x), f^{i}(y)\right)<\delta$ for $i=0,1, \cdots, n-1$. Suppose that $\Delta / 2$ is not an expansive constant for $f^{n}$. Then there exist distinct points $x$ and $y$, an orbit $A$ of $x$ under $f^{n}$ and an orbit $B$ of $y$ under $f^{n}$, such that for each integer $k, x_{k} \in A$ and $y_{k} \in B$ implies $d\left(x_{k}, y_{k}\right) \leqq \Delta / 2$. Let $m$ be any integer. Then there exist integers $i$ and $j$ such that $m=n j+i$, where $0 \leqq i \leqq n-1$. Define $x_{m}=f^{i}\left(x_{n j}\right)$ and $y_{m}=f^{i}\left(y_{n j}\right)$. Since $f^{i}\left(f^{n j}\right)=f^{m}$, the $x_{m}$ 's and $y_{m}$ 's define orbits under $f$ of $x$ and $y$ respectively. Also, $d\left(x_{m}, y_{m}\right)<\delta$ for each $m$, contradicting the assumption that $f$ is expansive with expansive constant $\delta$.

Lemma. If $f$ is a single-valued mapping of $[0,1]$ onto itself, then $f^{2}$ has at least two fixed points.

Received by the editors August 21, 1968. 
Proof. Certainly $f$ has at least one fixed point $x_{0}$. We may assume that $f(x)>x$ for $0 \leqq x<x_{0}$ and $f(x)<x$ for $x_{0}<x \leqq 1$, for otherwise $f$ and hence $f^{2}$ will have at least two fixed points. Hence, by onto-ness, there exists $x_{1} \in\left[0, x_{0}\right)$ such that $f\left(x_{1}\right)=1$ and there exists $x_{2} \in\left(x_{0}, 1\right]$ such that $f\left(x_{2}\right)=0$. By the intermediate value theorem, there exists $x_{3} \in\left(x_{0}, x_{2}\right]$ such that $f\left(x_{3}\right)=x_{1}$. Hence $f^{2}\left(x_{3}\right)=1 \geqq x_{3}$, and $f^{2}$ has a fixed point distinct from $x_{0}$.

THEOREM 2. If $f$ is a single-valued mapping of $[0,1]$ onto itself, then $f$ is not expansive.

Proof. Suppose that $f$ is expansive with expansive constant $\delta$. Using Theorem 1 and the preceding lemma, we may assume that $f$ has at least two fixed points. It is clear that $f$ can have only a finite number of fixed points, so let $a$ and $b$ be fixed with $b>a$, and let there be no fixed points between $a$ and $b$.

Case 1. Let $f(x)>x$ for $a<x<b$. If $f$ is monotone increasing on $[a, b]$, then for each $x \in(a, b)$, there exists an orbit $A$ of $x$ such that $x_{n} \in A$ implies $\lim _{n \rightarrow-\infty} x_{n}=a$ and $\lim _{n \rightarrow \infty} x_{n}=b$. It is therefore clear that there exist $x, y \in(a, b)$ with orbits $A$ and $B$ respectively such that for each integer $n, x_{n} \in A, y_{n} \in B$ implies $d\left(x_{n}, y_{n}\right)<\delta$. (This is essentially the proof of the nonexistence of an expansive homeomorphism on $[0,1]$ given in $[1]$.)

Suppose there exists $c \in(a, b)$ such that $f$ is monotone increasing on $[a, c]$, and that $c$ is the smallest such number. Then there exist distinct points $x$ and $y$, arbitrarily near $c$, such that $f(x)=f(y)$, and there exist orbits $A$ of $x$ and $B$ of $y$ such that $x_{n} \in A, y_{n} \in B, n<0$, implies $d\left(x_{n}, y_{n}\right)<\delta$. (Again, $f$ is a homeomorphism on $[a, c]$, and $\lim _{n \rightarrow-\infty} x_{n}$ $=\lim _{n \rightarrow-\infty} y_{n}=a$.)

Finally, suppose that $f$ is not monotone on any interval $[a, c]$, where $a<c<b$. Then, by repeated use of the intermediate value theorem, there exist distinct $x$ and $y$ in $[a, a+\delta]$, with orbits $A$ and $B$ respectively such that $f(x)=f(y)$, and $x_{n} \in A, y_{n} \in B, n<0$ implies $x_{n} \in[a, x], y_{n} \in[a, y]$. Thus $d\left(x_{n}, y_{n}\right) \leqq \delta$ for each $n$, again contradicting the expansiveness of $f$.

Case 2. Let $f(x)<x$ for $a<x<b$. Considering intervals of the form $[c, b], a<c<b$, the analysis of Case 1 can essentially be repeated, and the theorem follows.

The above theorem is not valid if one does not assume that $f$ is single-valued. Consider the following example:

Let $g$ be defined on $[0,1]$ by $g(x)=e^{2 \pi i x}$, and let $h$ be defined on the unit" circle by $h(z)=z^{2}$. Let $f$ be the (multi-valued) mapping of $[0,1]$ onto itself defined by $f(x)=g^{-1} h g(x)$. It is clear that $f^{n}(x)=g^{-1} h^{n} g(x)$ 
for $n=1,2, \cdots$, and it is also clear that the positive iterates of $h$ spread out distinct points on the unit circle. It follows that $f$ is an expansive mapping on $[0,1]$.

\section{BIBLIOGRAPHY}

1. B. F. Bryant, Expansive self-homeomorphisms of a compact metric space, Amer. Math. Monthly 69 (1962).

2. R. K. Williams, Expansive mappings, Amer. Math. Monthly 75 (1968).

SOUTHern Methodist University 